\title{
L'efficacité et l'équité des systèmes éducatifs sous \\ l'angle du curriculum prescrit dans une approche par compétence \\ Regard sur la situation belge francophone ${ }^{1}$
}

\section{Marc Demeuse, Natacha Duroisin, Sabine Soetewey et Antoine Derobertmasure}

À côté des recherches portant sur l'efficacité et l'équité des systèmes éducatifs à travers des comparaisons statistiques internationales ou l'étude des flux d'élèves au sein des systèmes éducatifs, il est possible de prendre en compte ces deux grandes dimensions dans le cadre de l'analyse des documents curriculaires. C'est ce que propose cet article dans un contexte très particulier, celui de la Belgique francophone où les programmes d'études, bien que cadrés par des textes communs, jouissent d'une très grande liberté dans leur conception. La thèse principale qui est développée ici postule que cette liberté, si elle existe bien, n'est sans doute pas un facteur favorisant efficacité et équité dans un système éducatif encore relativement peu régulé.

\section{Introduction}

Le système éducatif belge francophone est bien étudié sous l'angle de son efficacité et de son équité (Crahay, 2000; Demeuse, Baye, Straeten, Nicaise \& Matoul, 2005; Dumay \& Dupriez, 2009; Monseur \& Lafontaine, 2009). Ces deux dimensions sont à présent considérées non pas comme inconciliables, mais au contraire comme complémentaires (Demeuse, Crahay \& Monseur, 2001). Les travaux qui portent sur ce sujet sont généralement menés soit sous l'angle des résultats obtenus à des épreuves externes, comme dans les travaux menés à partir des données issues du Programme international pour le suivi des acquis des élèves (PISA) (Lafontaine \& Blondin, 2004; Baye et al., 2009) ou d'autres programmes internationaux, même si cette approche reste complexe à mener (Monseur \& Demeuse, 2004; Lafontaine et Monseur, 2012; Baye, Demeuse \& Friant, 2014), soit sous l'angle des flux d'élèves entre établissements et les sorties du système (Demeuse, Lafontaine \& Straeten, 2005; Malaise, Friant \& Demeuse, 2014). Les travaux que nous venons de mentionner conduisent malheureusement à une image assez peu positive, en regard de ces deux dimensions.

À côté de ces travaux, d'autres recherches, moins nombreuses, portent sur la conception des curriculums prescrits (Mangez, 2004; Dupriez, 2009; Mangez \& 
Mangez, 2008; Soetewey, Duroisin \& Demeuse, 2011; Bocquillon \& Derobertmasure, 2014; Duroisin \& Demeuse; 2015). Celles-ci peuvent, en partie au moins, éclairer les résultats précédents lorsque l'on s'intéresse à l'efficacité et/ ou à l'équité du système éducatif belge francophone. Par delà les spécificités du système éducatif belge francophone, nous espérons que le lecteur non spécialiste de ce système trouvera un intérêt par rapport à la démarche adoptée et les moyens de la transposer dans d'autres contextes.

\section{Comment analyser le curriculum en gardant en perspective l'efficacité et l'équité des systèmes éducatifs?}

\section{La Belgique: un (ou plusieurs) curriculum «national»?}

Il est sans doute un peu étrange de parler de "curriculums» ${ }^{2}$ sous une forme plurielle lorsqu'il s'agit d'un système éducatif. Il est néanmoins possible, dans le contexte qui sera étudié dans la suite de cet article, d'emprunter cette voie, tant ce qui est enseigné et même supposé être enseigné, si on se limite aux textes qui cadrent le travail enseignant, peut être différent. Ces différences se marquent non seulement d'une école à l'autre, mais même, au sein de celle-ci, d'une classe à l'autre, en fonction des options et des filières dans lesquelles ces classes s'inscrivent. Il ne s'agit donc pas ici de considérer les pratiques enseignantes comme source de diversité et, potentiellement, d'inefficacité ou d'iniquité, mais bien d'analyser les documents qui leur servent de base et qui, en principe, doivent les conduire à développer chez les élèves les compétences communes prescrites.

La Belgique présente une situation particulière en matière de curriculum. En effet, elle comporte à la fois trois systèmes extrêmement autonomes - il n'existe pas, en matière de curriculum, d'autorité commune à ces trois systèmes, ni même de lieu permanent de concertation entre eux - et à l'intérieur de ceux-ci, un grand nombre de structures publiques et privées subventionnées qui possèdent de très larges marges de manœuvre, y compris dans la définition des programmes d'études. Si d'autres pays connaissent également une juxtaposition de systèmes autonomes en matière d'éducation, comme en Suisse, par exemple, à travers les cantons, la Belgique présente la spécificité, à Bruxelles, d'une superposition, sur le même territoire, de deux systèmes (Communauté française et Communauté flamande) qui ne sont pas obligés de se parler alors même que les élèves, qui ne possèdent pas une "sous-nationalité» les rattachant nécessairement à l'une ou l'autre structure, peuvent changer d'école et donc passer d'un système à l'autre.

L'article 24 de la Constitution belge garantit en effet, depuis 1831, la liberté d'enseignement. Celle-ci s'applique aux parents (choix de l'établissement scolaire), mais aussi aux écoles qui jouissent d'une très large autonomie dans la manière dont elles organisent leurs enseignements. La Loi dite du «Pacte scolaire» (1959) est le garant de trois principes fondamentaux du système éducatif belge: 
la liberté de choix de l'école par les parents, la fin des tensions entre les réseaux d'enseignement publics et privés en terme de financement, notamment, et la gratuité de l'enseignement durant l'obligation scolaire, même si ce dernier principe reste encore incomplètement réalisé, comme le montre par exemple Bollens (2009). Cette loi a été votée avant la communautarisation de l'enseignement en 1989 et l'attribution des compétences en matière d'enseignement aux Parlements des trois communautés linguistiques du pays (Communauté flamande, Communauté germanophone et Communauté française, aujourd'hui baptisée «Fédération Wallonie-Bruxelles»). Depuis cette date, l'enseignement ne fait donc plus partie des prérogatives de l'État belge, devenu fédéral. Il n'y a dès lors pas, à proprement parler de curriculum national, mais au moins trois curriculums, un par communauté.

\section{La Communauté française de Belgique: un système bien complexe... et compliqué}

La Loi dite du «Pacte scolaire» a également permis de définir deux grands ensembles: les réseaux officiels et les réseaux libres subventionnés. Chacun de ces réseaux comprend des pouvoirs organisateurs différents, véritables autorités exerçant la responsabilité concrète d'organiser l'enseignement dans une ou plusieurs écoles. Dans les réseaux officiels, le pouvoir organisateur est toujours une personne de droit public, l'organisation de l'enseignement dit «officiel» est réalisée par le réseau de la Fédération Wallonie-Bruxelles (FWB) ou par le réseau des villes et des provinces. Il existe donc, sur le même territoire, plusieurs autorités publiques en charge des établissements scolaires publics. Celles-ci ne se partagent pas, comme dans d'autres systèmes, les responsabilités (par exemple, les bâtiments scolaires pour les municipalités, les départements et les régions, et les aspects pédagogiques pour l'État à travers les académies, comme en France), mais les exercent pleinement au sein des établissements qui en dépendent. Pour les réseaux libres subventionnés, le pouvoir organisateur est une personne de droit privé et l'organisation de l'enseignement dit «libre» est constituée d'un réseau confessionnel (majoritairement catholique) et d'un réseau non confessionnel, constitué d'écoles définissant leurs projets éducatifs et pédagogiques sur d'autres bases que religieuses (pédagogies principalement actives, se référant à des penseurs tels que Decroly, Freinet ou d'autres penseurs associés à ce courant). Certaines écoles libres subventionnées n'appartiennent à aucun réseau. À côté d'un enseignement formel, constitué en écoles réparties en réseaux et subventionnées par l'autorité publique (la Fédération Wallonie-Bruxelles, pour ce qui concerne cet article), les parents ont aussi la faculté d'instruire leurs enfants à domicile, sous le contrôle de l'inspection scolaire.

En Europe du Nord, le terme «curriculum» «est traditionnellement associé aux documents qui prescrivent les finalités, les objectifs et les contenus qui doivent être enseignés à un groupe particulier d'élèves et qui doivent être appris par eux durant leur cursus» (Westbury, 2007). Ceux-ci sont «publiés par les 
autorités nationales» (Sivesind, 2013). Si le système éducatif belge francophone dispose effectivement d'un "curriculum», il n'offre pas totalement «une vision d'ensemble, planifiée, structurée et cohérente des directives pédagogiques selon lesquelles organiser et gérer l'apprentissage en fonction des résultats attendus» (Demeuse \& Strauven, 2013, p. 11), la rédaction de chacun des programmes d'études relevant de la prérogative des différents réseaux d'enseignement et même, théoriquement, de chaque pouvoir organisateur. Pour rédiger leurs programmes d'études, ces derniers doivent prendre en considération des documents cadres (lois et décrets) et veiller à atteindre les exigences prescrites dans les Socles de compétences, au terme du premier degré de l'enseignement secondaire (grades 8 ), et dans les référentiels terminaux, au terme des $2^{\mathrm{e}}$ et $3^{\mathrm{e}}$ degrés de l'enseignement secondaire (grades 9 à 12). Ces documents cadres constituent, du point de vue des auteurs, le curriculum belge francophone. Il s'agit pourtant essentiellement d'une liste de compétences à atteindre alors que le curriculum ne se limite généralement pas à cela (Demeuse \& Strauven, 2013, p. 9).

Ce sont les programmes, propres à chaque réseau, voire à chaque pouvoir organisateur $^{3}$, qui précisent, entre autres, les finalités et valeurs, les objectifs, les méthodes pédagogiques, les matériels, les procédés d'évaluation pour mesurer l'atteinte des objectifs... dérivés des projets éducatifs et pédagogiques propres également à chacun des réseaux d'enseignement.

Selon l'article 5, $15^{\circ}$ du décret «missions» du 24 juillet 1997, qui cadre tout l'enseignement obligatoire en Belgique francophone, un programme d'étude est «un référentiel de situations d'apprentissage, de contenus d'apprentissage, obligatoires ou facultatifs, et d'orientations méthodologiques qu'un pouvoir organisateur définit afin d'atteindre les compétences fixées par le gouvernement pour une année, un degré ou un cycle». Ainsi, le réseau de la Fédération Wallonie-Bruxelles dispose des programmes qu'il définit, le réseau des villes et des provinces se réfere aux programmes des Provinces et Communes et les réseaux libres appliquent leurs propres programmes. Concrètement, pour une année d'étude donnée (dans une filière identique, de même forme et option) appartenant à deux réseaux d'enseignement différents, le contenu de chaque cours est fixé par des programmes différents, puisque rédigés de façon autonome par chaque réseau, dans le respect du référentiel commun. Les programmes sont soumis pour avis, par chaque pouvoir organisateur, à la commission de programme correspondant au niveau et type d'enseignement concerné ${ }^{4}$. Ces commissions sont notamment composées de membres de l'inspection et de représentants des pouvoirs organisateurs qui y sont majoritaires. Leur avis est transmis pour approbation au Ministre en charge de l'enseignement obligatoire qui prend aussi l'avis (1) de la Commission de pilotage, instance composée des mêmes catégories de membres auxquels s'adjoignent des représentants des associations de parents, des syndicats d'enseignants, de l'administration de l'enseignement et des chercheurs en éducation, (2) des Conseils généraux de concertation compétents et (3) des organisations syndicales. Comme le précise le décret «missions», «la Commission 
des programmes vérifie si les programmes d'études sont de nature à permettre la maîtrise des acquis d'apprentissage fixés» par les référentiels communs, mais le texte poursuit en indiquant que «le contrôle de la Commission des programmes ne porte pas sur les méthodes pédagogiques». Comme le montre une lecture des textes normatifs, les pouvoirs organisateurs qui développent les programmes sont aussi très largement impliqués dans l'agrément de ceux-ci par le ministre compétent.

\section{Une complexité qui conduit à bien des problèmes}

Le principe de liberté d'enseignement conduit inévitablement à une pluralité dans l'approche de ce qui doit être enseigné et pas seulement dans la manière dont il doit l'être. Étant donné que chacun des réseaux rédige ses propres programmes pour chaque niveau d'enseignement (enseignement maternel, enseignement primaire, enseignement secondaire de transition, enseignement secondaire de qualification et enseignement secondaire professionnel) et les différentes disciplines (mathématiques, français, sciences, géographie, éducation physique, ...), le nombre de programmes d'études disponibles et utilisés pour l'enseignement obligatoire est très important. Le découpage des disciplines peut lui aussi varier d'un réseau à l'autre (par exemple, un cours d'histoire et un cours de géographie distincts dans l'enseignement officiel pour le premier degré de l'enseignement secondaire, mais un seul cours d'étude du milieu pour l'enseignement libre catholique).

La multitude des programmes d'études peut donc être envisagée du point de vue de la recherche en mettant l'accent sur l'évaluation de leur cohérence pour conduire à la maîtrise d'un curriculum commun. Comme nous avons déjà pu le montrer dans d'autres articles (Soetewey et al., 2011; Duroisin, 2015), une part des échecs scolaires (notamment aux enquêtes internationales telles que le PISA et aux évaluations externes non-certificatives réalisées en Belgique francophone) peut être due, notamment, à un ensemble d'incohérences dans la mise en œuvre du curriculum commun. Cela se marque aussi bien en termes de rendement moyen (efficacité) que d'accroissement des écarts entre élèves, principalement en fonction de leurs caractéristiques socio-économiques et de leurs parcours scolaires (équité).

En effet, cette multitude de référentiels amène les enseignants des différents réseaux à évaluer des apprentissages similaires à différents moments de la scolarité (Soetewey et al., 2011), alors même que les élèves peuvent changer de réseau, mais aussi de filière ou d'option, durant les quatre dernières années de l'enseignement obligatoire. Ce manque de cohérence s'est cristallisé dans le contexte de la mise en place d'un dispositif d'évaluation externe non certificatif des compétences en sciences (EENC 5 ), lorsqu'un accord, entre les différents réseaux, sur la portée de l'évaluation à un moment donné de la scolarité n'a pu être trouvéf. C'est donc principalement à travers les programmes de sciences que nous poursuivrons notre analyse, même si nous avons étudié d'autres domaines. 
Au-delà du problème général que nous venons d'évoquer, l'analyse des référentiels et des programmes de cours en sciences met en évidence des incohérences dans l'usage même de la notion de compétence, confondant, dans ce qui est prescrit au sein des programmes, des savoirs, des savoir-faire et des compétences de degrés divers de complexité (Duroisin, Soetewey \& Demeuse 2012; Duroisin, 2015). Le rôle de modèle, assigné aux référentiels de compétences, perd ici de sa consistance ce qui entrave l'appropriation de la notion de compétence et, de fait, sa transposition dans l'évaluation des acquis des élèves par les enseignants. Ces incohérences affaiblissent la compréhension que les enseignants ont de cette notion sur l'évaluation des compétences (Soetewey, Demeuse, Duroisin, Letor \& Malaise, 2014 et «Des usages «officiel » approximatifs et des enseignants parfois fort perdus face aux «concepts-clés» des référentiels» de l'article.

\section{L'entrée par les compétences ne simplifie donc pas la tâche des enseignants... et accroît encore le risque de dérégulation}

\section{Des définitions multiples et peu opérationnelles du concept de compétence}

L'entrée actuelle dans le curriculum est officiellement une entrée par les compétences et ce concept-clé du système éducatif belge francophone n'est pas sans poser problème. Bien d'autres (par exemple, Hirtt, 2005; Crahay, 2006; Schneider-Gilot, 2006; Beckers \& Voos, 2008; Legendre, 2008), parfois de façon virulente ou polémique, l'ont fait remarquer avant nous (Duroisin, Soetewey $\&$ Demeuse, 2012). L'entrée par les compétences ${ }^{7}$ complique singulièrement la tâche des enseignants, peu à l'aise avec le concept lui-même ou plutôt avec ses implications pédagogiques, même si ce concept fait partie du paysage belge francophone depuis au moins 1997 et le décret «missions» ${ }^{8}$. Contrairement au terme "compétence», défini par ce texte officiel comme «l'aptitude à mettre en œuvre un ensemble organisé de savoirs, de savoir-faire et d'attitudes permettant d'accomplir un certain nombre de tâches» (article 5), les éléments constitutifs de cette définition ne sont définis nulle part, ni dans le décret, ni, par exemple, dans les programmes d'études que nous avons analysés, et aucune référence n'est fournie à leur sujet, comme si cela allait de soi. À côté de la définition officielle de la compétence, d'autres auteurs francophones, belges ou étrangers, ont proposé des définitions, comme Tardif (2006, p. 22) qui la définit comme «un savoir-agir complexe prenant appui sur la mobilisation et la combinaison efficaces d'une variété de ressources internes et externes à l'intérieur d'une famille de situations». Cette définition ajoute à la définition décrétale d'autres composantes, comme cette référence à la complexité ou l'idée de famille de situations. De nombreux autres auteurs ont aussi attaché au concept de compétence, un caractère global, évolutif ou inédit (Beckers, 2002; Depover \& Noël, 2005; Legendre, 2004; 
Perrenoud, 1999; Roegiers, 2000). Certains référentiels font aussi appel à d'autres concepts, comme les «macro-compétences» dans l'enseignement catholique (Hirtt, 2005) ou à des compétences qualifiées de disciplinaires, voire spécifiques, ou de transversales. Face à cette abondance de définitions et de concepts, il est assez difficile pour chaque enseignant de bien se représenter ce qu'est finalement une compétence à faire acquérir, à exercer en classe ou à évaluer.

\section{Des usages "officiels" approximatifs et des enseignants parfois fort perdus face aux "concepts-clés» des référentiels}

Au-delà même de la définition formelle des concepts-clés que nous avons abordés jusqu'ici, l'analyse des référentiels communs à tous les réseaux d'enseignement (compétences terminales et savoirs requis en sciences pour les humanités générales et technologiques) et des programmes d'études du réseau de la Communauté française de Belgique fait apparaitre la grande confusion qui règne dans l'usage pratique qui est fait des termes "compétence», «savoir», «savoir-faire» ou encore «attitude». Ainsi, sous le vocable de "compétence spécifique», on retrouve dans le référentiel commun de fin d'enseignement secondaire ce qui nous semble être des savoir-faire comme "utiliser une clé dichotomique», "utiliser un multimètre» ou «représenter dans le plan une molécule d'hydrocarbure saturée» (ce dernier énoncé pouvant sans doute même être assimilé à un savoir) ou des savoirs, comme "connaître les principes de fonctionnement du corps humain» ou «savoir (sic) que la croissance, l'émotivité, les cycles sexuels sont sous la dépendance d'hormones» (Duroisin, Soetewey \& Demeuse, 2012).

Il n'est dès lors pas étonnant que les 23 enseignants 9 que nous avons interrogés de manière à mieux comprendre comment les programmes sont reçus par ceux à qui ils sont d'abord destinés, définissent de manière parfois très hésitante et très peu satisfaisante ces différents concepts, pourtant fondamentaux par rapport à leur propre métier, comme l'indiquent les deux définitions suivantes de «un savoir»:

Extrait 1: "Le savoir, ce sont les choses qu'ils doivent, euh, quand même, euh, et de un, euh, connaître au départ, et, pouvoir, éventuellement, les améliorer.»

Extrait 2: "Un savoir? Ben c'est à partir du moment où il a compris, je vais dire, compris, où il a su faire le lien entre ce qu'il a appris en classe et la pratique, parce que vous savez, vous apprenez également à bien connaître le code de la route, si vous conduisez la voiture comme un sauvage, ça sert à rien..."

Mais, la définition d'un savoir-faire n'est pas plus aisée, comme l'indique cet autre enseignant:

Extrait 3: "Euh jrai plus facile à travailler un savoir-faire parce que c'est, pour moi c'est du conditionnement, donc je leur montre comment ça se passe, je leur donne un exercice quills font avec moi, au fur et à mesure, et puis la deuxième 
fois, je les laisse se tromper, donc je passe entre les bancs, ça ça va, ça ça va pas et la troisième fois, je commence à vérifier et à vraiment commencer à noter... Et donc après, je les drille, devoir sur devoir, et ça passe..."

Concernant le terme "compétence», les explications recueillies chez les 23 enseignants déjà évoqués se réferent à trois modèles distincts. Le premier d'entre eux est celui présenté par le décret "missions» 10 et complété par le message des conseillers pédagogiques qui mentionnent le caractère inédit et «non-pédagogique» de la tâche. Le deuxième porte sur les seuls éléments énoncés par le décret «missions». Le troisième et dernier modèle évoqué est celui de la compétence comme «intersection de trois cercles représentant les savoirs, savoir-faire et attitudes». À côté de ces trois types de réponses, en rapport avec la définition légale ou des modèles utilisés lors de formations officielles, on trouve aussi des réponses beaucoup plus confuses, comme les deux suivantes:

Extrait 4: «Ben c'est, c'est, c'est la ligne de conduite principale à tenir, c'est le contenu principal de la matière, que liélève doit comprendre, c'est le franc qui doit tomber..."

Extrait 5: "C'est d'arriver à inculquer à un élève une matière, euh (Petite pause), et qu'il puisse la trouver, pas lui donner et lui imposer, c'est qu'il puisse, euh, après, euh, diverses choses arriver à, par lui-même à la trouver."

Les définitions proposées sont aussi assorties de certains doutes ou remarques qui indiquent bien la confusion qui existe autour du terme "compétence», comme le montrent les extraits suivants:

Extrait 6: "(...) [Dans] ce bouquin «socle de compétences», ce ne sont pas des compétences. Et dans le programme, on a la même chose et donc on est en désaccord, on utilise un même terme pour deux choses tout à fait différentes, [...] euh compétences en math, compétences en chimie, en physique, en bio, compétences en français ou en langues, c'est le même mot pour des choses totalement différentes..."

Extrait 7: "Donc comment voulez-vous libeller tout ça? Nous, entre collègues de sciences, on estime que c'est impossible à libeller... Donc quand les collègues le font, (...) ils ne font que libeller un savoir-faire (...)»

Extrait 8: "Les compétences, il nyy a aucune compétence, enfin diaprès ce que jrai compris, il n'y en a aucune dans le programme hein. Les compétences c'est quelque chose qu'on crée. (...) on utilise beaucoup le terme "compétence», mais euh en tant que prof, je m'emmêle un peu les pinceaux. On me parle de compétences, de savoirs, de savoir-faire, et de motivations d'élève, au milieu c'est la compétence (...) je suis un peu perdue moi».

Extrait 9: "Voilà, je vais vous traduire ce quıon m'a expliqué et que j'ai, enfin je pense, avoir accepté, je pense..." 
Extrait 10: (Pause). "On se dit mais 'Qu'est-ce qu'ils nous veulent?'».

\section{Extrait 11: "En fait, ça dépend, c'est très difficile à définir, une compétence.»}

Extrait 12: "J'espère que je ne me trompe pas, parce que bon, c'est toujours difficile»

Dans le but de constater si les enseignants maitrisent les concepts-clés issus des programmes d'études, cet échantillon d'enseignants a été confronté à une série d'énoncés qu'ils ont dû identifier comme étant des "savoirs", des "savoir-faire», des "compétences» ou d'autres choses. Seize énoncés, provenant des programmes d'études en sciences, ont été sélectionnés pour cet exercice. Ces programmes identifient 9 de ces énoncés comme étant une compétence et 7 autres comme un savoir. L'équipe de recherche a, quant à elle, analysé ces mêmes énoncés et compte parmi eux 3 compétences, 9 savoirs, 3 savoir-faire. Un énoncé ne semble appartenir à aucune de ces catégories. À l'issue de l'exercice, il apparaît que l'identification effectuée par les enseignants est plus proche de celle réalisée par l'équipe de recherche que celle provenant des référentiels. Sans grande surprise, le degré de confusion lors de l'exercice de définition a un impact important sur l'identification de la nature des énoncés par les enseignants.

\section{Alors, qu'évalue-t-on?}

Contrairement à l'adage d'Ovide Decroly qui veut que «tout ce qui doit être enseigné par le maître, donc appris par l'élève, c'est-à-dire, tout ce qui est matière d'instruction, est susceptible de mesure» (Decroly \& Buyse, 1929, p. 11), on peut être surpris par certains énoncés qualifiés de "compétence» dans les référentiels et dont il est difficile d'imaginer sérieusement comment les enseignants en vérifieraient la maîtrise, en dehors de déclarations assez générales... démontrant certainement plus un certain savoir qu'une réelle compétence. Il en va ainsi de la surprenante "compétence mise en œuvre» intitulée «avoir une attitude responsable en face de la sexualité: pouvoir gérer ses pulsions éventuelles» inscrite dans le "programme provisoire [et toujours disponible] d'études du cours de sciences (formation scientifique et technologique) du troisième degré de l'enseignement secondaire ordinaire de plein exercice (humanités professionnelles et techniques» ${ }^{11}$ (page 50), dans un module consacré à la reproduction chez l'homme ${ }^{12}$.

Plus sérieusement, serions-nous tentés de dire, si cette référence ne figurait dans des recommandations officielles, comment les enseignants doivent-ils concevoir leurs cours et... les évaluations à partir d'énoncés qui semblent fort imprécis? C'est aussi ce que se pose comme question Hirtt, en 2005, à propos de l'enseignement de la physique dans le réseau catholique cette fois:

Dans l'OG [option groupée] «Sciences appliquées», le programme de l'enseignement catholique mentionne, pour les «contenus obligatoires» du chapitre «électromagnétisme» au $3 e$ degré: "champ magnétique, force électroma- 
gnétique, courants induits». C'est tout! Six mots seulement pour décrire le contenu de ce qui, sur base de mon expérience des anciens programmes, occupe les élèves de sixième durant au moins trois mois, à raison de trois heures par semaine.

Or, le sujet est complexe et permet bien des interprétations personnelles. Comment dois-je aborder le concept de champ magnétique? [..] On n'en sait rien. On supposera qu'il faut aborder la question du magnétisme de la matière (quoique le programme n'en fasse pas mention), mais jusqu'à quel niveau de détail? Mystère!

Cette incertitude sur ce qu'il faut enseigner et jusqu'où il faut l'enseigner se double naturellement d'une incertitude sur ce qu'il faut évaluer et jusqu'où il faut l'évaluer. Sans autres précisions, on peut partager les craintes de Hirtt (2005) par rapport à une dérégulation du système éducatif qu’il décrit ainsi:

Les enseignants ne manqueront pas de remplir eux-mêmes les trous. Ils se chargeront de donner du corps aux définitions sibyllines des "contenus obligatoires». Mais ils le feront forcément en adaptant spontanément leurs exigences au niveau du public de leur classe. Or, cette bonne volonté conduira au résultat inverse de la justice recherchée. Une fois de plus, la somme de bonnes volontés individuelles, dans un système basé sur la concurrence et la compétition, conduira vers l'augmentation des inégalités. Les savoirs complexes et les développements mathématiques de haut niveau seront réservés aux écoles de l'élite sociale; les autres développeront leurs «macro-compétences»-bidon sur des chantiers de problèmes simplistes en y mobilisant des savoirs superficiels.

S'il peut sembler normal que les enseignants adaptent jusqu'à un certain point les programmes localement, l'absence de précision, liée à une adoption sans doute trop radicale de l'entrée par les compétences, facilite la dérive du curriculum, de manière d'autant plus aisée qu'il n'existe, par exemple, ni manuels communs et obligatoires, ni évaluations externes certificatives à tous les moments-clés du parcours. Ces absences sont aussi renforcées par la présence d'un quasi-marché scolaire (Dupriez \& Dumay, 2011), d'une importante concurrence entre établissements scolaires et d'un marché de niche conduisant à une très forte ségrégation des publics scolaires à la fois sur une base académique et socio-économique (Demeuse \& Baye, 2007, 2008; Demeuse \& Friant, 2010). Le système éducatif belge francophone, extrêmement dualisé, comme l'indiquent les résultats aux enquêtes PISA, se nourrit ainsi de référentiels multiples et imprécis, fruit d'une liberté d'enseignement d'un autre âge. 


\section{Cohérence interne et continuum pédagogique}

D'autres pistes peuvent encore être empruntées pour traquer les sources possibles d'inefficacité et d'iniquité, comme nous l'avons notamment fait dans un article récent (Duroisin \& Demeuse, 2015). Cet article et la recherche qui le sous-tend privilégient une autre approche de l'évaluation de la cohérence du curriculum: il s'agit de vérifier la cohérence interne des programmes et le continuum pédagogique proposé en regard d'un modèle développemental donné au sein d'un même réseau d'enseignement. À l'intérieur même des programmes d'études d'un réseau d'enseignement peuvent être identifiées des incohérences par rapport au développement psycho-cognitif de l'enfant, ce qui constitue une source possible d'échecs scolaire. Cette analyse a été menée dans plusieurs domaines. Elle l'a été en particulier dans le domaine de la géométrie pour les documents couvrant la période de scolarité primaire et le début de l'enseignement secondaire, soit neuf des douze années de l'enseignement obligatoire.

Globalement, l'analyse menée met en évidence que les programmes d'études se basent peu sur des théories développementales spécifiques au domaine de la géométrie (telle que celle proposée par Van Hiele, 1986) et celles-ci ne sont pas explicitées à destination des enseignants. Si ces programmes d'études privilégient de manière quasi-exclusive, durant l'enseignement primaire, le travail sur des objets concrets, ils prennent peu en compte les récentes recherches qui mettent en évidence l'intérêt de débuter le processus d'abstraction dès le troisième cycle de l'enseignement primaire. Ils négligent tout autant l'intérêt des manipulations dans l'enseignement secondaire. Pour les futurs programmes d'études, il parait utile de penser, à priori, lors de la rédaction, à intégrer les apports de ces nouvelles recherches (Duval, 2005; Mathé, 2008; Perrin-Glorian, Mathé \& Leclercq, 2013).

Si bon nombre de documents cadres et de nombreuses recherches tentent de résoudre les difficultés rencontrées lors de la transition primaire/secondaire, il semble aussi nécessaire de ré-écrire les programmes en étant attentif aux acquis antérieurs des élèves, favorisant ainsi le développement d'un continuum cohérent. C'est d'ailleurs la différence de résultats entre les deux seules évaluations externes certificatives - celle de fin d'enseignement primaire et celle de fin d'enseignement secondaire inférieur - rendues obligatoires très récemment (juin 2009, pour la première, juin 2014, pour la seconde) qui a, notamment, conduit à s'interroger sur l'existence d'un réel curriculum cohérent, notamment en géométrie (Usiskin, Andersen \& Zotto, 2010; Houdement, 2007; Duroisin, 2015).

Comme nous le signalions dans le travail réalisé par rapport aux programmes de sciences du secondaire supérieur, le manque de précision dans la rédaction des programmes de mathématiques, partie géométrie, et le manque d'illustrations risque d'induire une importante dérive curriculaire, en fonction du niveau des élèves et des disparités importantes observées entre établissements scolaires 
(Soetewey et al., 2011; Duroisin \& Demeuse, 2014). Cette difficulté est encore plus importante pour les enseignants du secondaire qui ne disposent pas nécessairement de la formation initiale adéquate et donc du titre requis pour enseigner les mathématiques à ce niveau (Duroisin, 2015).

\section{Formation initiale des enseignants et programmes multiples: comment s'y prendre sans s'y perdre ou s'épuiser?}

Une piste que les auteurs n'ont pas encore empruntée et pour laquelle ils n'ont pu identifier de littérature scientifique spécifique concerne la manière dont les futurs enseignants s'approprient ces programmes durant leur formation initiale et comment leurs formateurs utilisent ceux-ci. En effet, si les programmes sont amenés à se modifier à travers le temps, comme dans tout système éducatif, il faut aussi considérer la situation particulière des enseignants belges francophones lors de leur recrutement: ceux-ci sont formés dans les institutions d'enseignement supérieur, au sein des universités ou en dehors de celles-ci (Hautes Écoles, Écoles supérieures des Arts et Écoles supérieures de Promotion sociale), appartenant aux différents réseaux d'enseignement présents dans l'enseignement obligatoire, mais ne sont pas nécessairement recrutés par les établissements d'enseignement des réseaux correspondant à celui de leur formation initiale. Il est dès lors très difficile de prévoir, lors de cette formation, les programmes auxquels les futurs enseignants seront confrontés dès leur entrée dans le métier. Il est par ailleurs impossible, compte tenu du nombre de programmes en vigueur, de former chaque futur enseignant à la maîtrise de chaque programme. Comment alors penser la formation initiale? Comment faire découvrir les programmes qui seront utilisés durant les premières années en service et même, comment préparer les stages qui peuvent se dérouler dans les différents réseaux d'enseignement et avec des programmes différents? Est-il possible de faire fi des programmes spécifiques (mais alors, à quoi bon ces programmes distincts)? Est-il possible d'aborder les programmes de manière suffisamment abstraite pour les envisager tous (mais alors comment rendre les choses assez abordables pour des novices)? Ce sont autant de questions que nous nous posons tous les jours lorsque nous sommes responsables de la formation de nos propres étudiants et nous sommes encore aujourd'hui très surpris de ne pas trouver d'éléments de réponse dans la littérature alors même que tous les formateurs d'enseignants en Belgique francophone sont confrontés au même problème... sauf à faire comme si cela n'existait pas ou comme si les programmes n'avaient aucune importance. 


\section{Conclusion}

L'analyse des référentiels (curriculum prescrit) permet de comprendre à priori certains résultats (curriculum maîtrisé) et le fonctionnement du système (curriculum implanté) sans même devoir se rendre en classe, à moins naturellement de considérer qu'ils ne sont pas utilisés par les enseignants ou qu'ils ne sont pas destinés à être mis en pratique par ceux-ci.

Les incohérences qui ont été constatées lors d'une série de recherches menées par les auteurs, principalement dans le domaine de l'enseignement des sciences dans le secondaire supérieur, permettent d'interroger le système éducatif sur sa véritable volonté de réguler les apprentissages, au-delà même de la très grande liberté qui est accordée aux différentes structures intermédiaires que constituent les réseaux d'enseignement. À la lecture, et surtout à l'analyse fine des textes qui constituent les programmes d'enseignement et les référentiels communs, semble bien se dégager la primauté de la liberté sur la volonté d'efficacité et d'équité.

La conception des référentiels renforce encore les autres mécanismes (par exemple, la ségrégation en augmentant l'inégale valorisation des filières et la non réversibilité des parcours). La "liberté de choix», constitutive du système éducatif belge francophone, est principalement favorable à ceux qui se maintiennent dans un parcours sans bifurcation et au sein de la même structure, même si la cohérence des programmes au sein d'un même réseau d'enseignement est déjà questionnable, ne serait-ce qu'à la transition entre l'enseignement primaire et l'enseignement secondaire inférieur ou entre ce dernier et le secondaire supérieur.

Les enseignants des filières professionnelles, considérées assez unanimement comme des filières de relégation qu'il conviendrait de revaloriser (Franquet, Friant $\&$ Demeuse, 2010; Friant \& Demeuse, 2012), sont ceux qui sont confrontés à la plus forte hétérogénéité des parcours et donc des compétences des élèves. Il est dès lors particulièrement difficile pour eux d'atteindre les objectifs qui sont assignés à ces filières. L'article 10 du décret «missions» proscrit cependant toute mesure susceptible d'instaurer une hiérarchie entre établissements ou entre sections et formes d'enseignement au sein de l'enseignement secondaire. On le voit, malgré cette déclaration de principe, les bases de cette hiérarchisation sont bien présentes dans les programmes d'études. Penser les parcours, les rendre possibles, sans trop de redites ou d'oublis, et surtout les rendre lisibles en évitant que les filières de qualifications ne représentent qu'une dégradation/appauvrissement des programmes de l'enseignement général, c'est sans doute ce qui constitue une véritable «approche programme» (Prégent, Bernard \& Kozanitis, 2009).

L'entrée par les compétences rend plus complexe encore la compréhension des référentiels et augmente la possibilité d'interprétations divergentes. Le manque de cohérence des référentiels augmente aussi cette possibilité et, de ce fait, les dérives du curriculum (baisse des exigences face aux élèves jugés plus faibles... 
d'autant plus qu'ils sont regroupés dans un système fortement ségrégué) sont d'autant plus difficiles à éviter.

Il est donc essentiel de proposer des programmes de qualité "professionnelle» qui présentent au moins une forte cohérence (Jonnaert, Ettayebi \& Defise, 2009; Demeuse \& Strauven, 2013). La manière dont ils sont élaborés, de même que les référentiels communs sur lesquels ils doivent s'appuyer, mériterait d'être très largement revue. On ne peut confier à des groupes d'enseignants, même de très bonne volonté, la seule responsabilité d'écrire les programmes. En ce qui concerne les référentiels, il ne s'agit plus, à présent, de faire consensus autour de documents assez flous pour permettre à chacun de maintenir ses propres programmes, mais bien de développer une véritable épine dorsale pour les apprentissages jugés nécessaires, compte tenu des connaissances scientifiques en matière de didactique, mais aussi de psychologie développementale (Duroisin, 2015; Duroisin, Soetewey \& Canzittu, 2013). Ces informations doivent également être communiquées aux enseignants qui devront s'y référer de manière à leur permettre de comprendre ce qui est réellement attendus d'eux, comme professionnels.

L'offre d'enseignement doit, elle aussi, être rationalisée et orientée vers les élèves et non vers les structures existantes, le maintien des parts de marché ou les situations historiques. Si on peut entendre la volonté de préserver certaines marges de liberté dans l'élaboration des programmes, on comprend mal, pour rester dans le domaine des sciences qui a fait l'objet d'une majorité de nos travaux, pourquoi il serait impossible de fixer des objectifs communs plus précis à des niveaux plus resserrés de manière à tenir compte de la mobilité réelle des élèves entre écoles et réseaux d'enseignement, ce qui constitue pourtant le fondement de la liberté d'enseignement dont se réclament généralement les réseaux eux-mêmes.

\section{Notes}

1 Ce texte a servi de base à une conférence donnée lors du Congrès 2014 de la Société suisse pour la recherche en éducation (SSRE) à Lucerne en juin 2014. Il propose une synthèse de certains travaux menés au sein de l'Institut d'Administration scolaire de l'Université de Mons (UMONS) dans le domaine de l'étude des curriculums. Une partie de ces travaux a été rendue possible grâce à des subventions du Ministère de la Communauté française de Belgique et à un financement du F.R.S.-FNRS.

2 Nous avons préféré le pluriel "curriculums» à sa forme latine "curricula», suivant en cela l'option que nous avions prise dans un ouvrage antérieur (Demeuse \& Strauven, 2013) ou la voie choisie par Audigier et Tutiaux-Guillon (2008).

3 À titre d'information, le site officiel de la Communauté française ne recense pas moins de 7 ensembles de programmes différents et approuvés, rien que pour l'enseignement primaire ou certaines disciplines au sein de celui-ci (http://www.enseignement.be/index. php?page=0\&navi=2438, consulté le 26 décembre 2014), soit un pour le réseau de l'Enseignement organisé par la Fédération Wallonie-Bruxelles, un pour le Conseil de l'Enseignement des Communes et des Provinces (CECP), un pour la Fédération de l'Enseignement Libre Subventionné Indépendant (FELSI), un pour le Secrétariat général de l'Enseignement Catholique, un pour la Libre École Rudolph Steiner et deux autres relatifs à des villes, pourtant membres du CECP: Verviers et Bruxelles. Certains programmes sont 
composés de plusieurs documents distincts, approuvés à des moments différents et, à titre d'illustration, le programme de la ville de Bruxelles reprend celui du CECP, à l'exception des parties relatives à l'enseignement du français et des mathématiques.

4 Il existe quatre commissions de programmes, l'une pour l'enseignement fondamental et le 1er degré de l'enseignement secondaire, une pour les humanités générales et technologiques, une pour les humanités professionnelles et techniques et une pour l'enseignement secondaire spécialisé (source: http://www.enseignement.be/index.php?page=25279, site officiel de la Communauté française, consulté le 26 décembre 2014)

5 Évaluations externes non certificatives destinées au pilotage du système éducatif.

6 Il s'agit en particulier de l'impossibilité qui a été constatée par la Commission de pilotage de créer une évaluation externe non certificative à l'issue du grade 10 (4e année de l'enseignement secondaire) en sciences. C'est précisément à ce niveau que se trouvent les élèves de 15 ans à l'heure, ce qui constitue en principe l'âge de référence pour l'échantillon PISA.

7 Pour une discussion à propos de l'entrée par les compétences, on peut par exemple consulter Demeuse \& Strauven (2013, pp. 75-97).

8 Il existait même, avant ce décret, une première mouture des socles de compétence, produite par le cabinet du Ministre Mahoux (1994) alors que les Socles actuels datent de 1999.

9 Dans le cadre d'une recherche qualitative dont le but était d'appréhender la perception et les difficultés des enseignants utilisant les programmes d'études, des questionnaires ont été distribués à un échantillon de convenance de 23 enseignants issus des degrés 2 et 3 (grades 9 à 12) de l'enseignement secondaire du réseau d'enseignement organisé par la Fédération Wallonie-Bruxelles. Ces enseignants ont également répondu à une interview définie selon un canevas en entonnoir, faisant se succéder entretiens non dirigé et semi dirigé, puis des questions fermées (Duroisin, Soetewey \& Demeuse, 2012, Duroisin, 2015). L'analyse qualitative des informations a été réalisée à l'aide du logiciel NVivo9.

10 "Compétence: aptitude à mettre en œuvre un ensemble organisé de savoirs, de savoirfaire et d'attitudes permettant d'accomplir un certain nombre de tâches» (Définition de la compétence selon le décret «missions» du 24 juillet 1997).

11 Référence: Ministère de la Communauté française, Enseignement de la Communauté française, Administration générale de l'Enseignement et de le Recherche scientifique, Service général des Affaires pédagogiques, de le Recherche en Pédagogie et du Pilotage de l'Enseignement organisé par la Communauté française, nº 123/2002/248 B, année 2002, Récupéré le 26 décembre 2015 de http://www.restode.cfwb.be/download/ programmes/123-2002-248B.pdf

12 Le lecteur sera encore plus intrigué par la mention qui est faite dans la rubrique «exemples d'activités» de la même fiche: «organiser des manipulations en relation avec le module»!

\section{Bibliographie}

Audigier, F. \& Tutiaux-Guillon, N. (Éd.). (2008). Compétences et contenus. Les curriculums en questions. Bruxelles: De Boeck.

Baye, A., Demeuse, M. \& Friant, N. (2014). Comparer les systèmes éducatifs francophones à travers le monde grâce au PISA: pas si simple! Éducation et francophonie, Volume XLII: 3, 102-122. Consulté le 26 décembre 2014 dans http://www.acelf.ca/c/revue/pdf/EF42-3NoSpecial-102-BAYE-DEMEUSE.pdf.

Baye, A., Fagnant, A., Hindryckx, G., Lafontaine, D., Matoul, A. \& Quittre, V. (2009). Les compétences des jeunes de 15 ans en Communauté française en sciences, en mathématiques et en lecture. Résultats de l'enquête PISÀ 2006. Liège, Belgique: Université de Liège - analyse des Systèmes et Pratiques d'enseignement. Consulté le 26 décembre 2015 dans http://orbi. ulg.ac.be/bitstream/2268/19520/1/CAHIERS\%20PISA\%2029-30\%20.pdf.

Beckers, J. (2002). Développer et évaluer des compétences à l'école: vers plus d'efficacité et d'équité. Bruxelles: Labor. 
Beckers, J. \& Voos, C. (2008). Savoirs scolaires et compétences, les difficultés de cadrage d'une réforme en Communauté française de Belgique. In F. Audigier, N. TutiauxGuillon (Éd.), Compétences et contenus. Les curriculums en questions (pp. 51-64). Bruxelles: de Boeck.

Bocquillon, M. \& Derobertmasure, A. (2014). Étude comparative des programmes de français des réseaux catholiques et de la Fédération Wallonie-Bruxelles pour le premier degré de l'enseignement secondaire. Éducation comparée, 11, 217-238.

Bollens, J. (2009). Venir à bout des barrières financières. In I. Nicaise, E. Desmedt \& M. Demeuse (Éd.), Une école réellement juste pour tous! Seize pistes de réforme et d'action (pp. 107-130). Watterloo: Plantyn.

Crahay, M. (2000). L'école peut-elle être juste et efficace? Bruxelles: De Boeck Université.

Crahay, M. (2006). Dangers, incertitudes et incomplétude de la logique de la compétence en éducation. Revue française de pédagogie, 154, 97-110.

Decroly, O, Buyse, R. (1929). Introduction à la pédagogie quantitative. Éléments de statistique appliqués aux problèmes pédagogiques. Bruxelles: Maurice Lamertin.

Demeuse M. \& Baye A. (2007), Measuring and Comparing the Equity of Education Systems in Europe. In N. C. Soguel, \& P. Jaccard (Ed.), Governance and Performance of Education Systems (pp. 85-106). Dordrecht: Springer.

Demeuse, M. \& Baye, A. (2008). Indicateurs d'équité éducative. Une analyse de la ségrégation académique et sociale dans les pays européens. Revue Française de Pédagogie, 165, 91-103.

Demeuse M. \& Friant N. (2010), School segregation in the French Community of Belgium. In J. Bakker, E. Denessen, D. Peters, \& G. Walraven (Ed.), International perspectives on countering school segregation (pp. 173-192). Apeldoorn: Garant.

Demeuse, M. \& Strauven, C. (2013, 2e édition). Développer un curriculum d'enseignement ou de formation. Des options politiques au pilotage. Bruxelles: De Boeck Université.

Demeuse, M., Crahay, M. \& Monseur, C. (2001). Efficiency and Equity. In. W. Hutmacher, D., Cochrane, \& N. Bottani (Ed.), In pursuit of equity in education: using international indicators to compare equity policies (pp. 63-91). Dordrecht: Kluwer Academic Publishers.

Demeuse, M., Duroisin, N. \& Soetewey, S. (2012). Implications du choix des référentiels dans les évaluations nationales et internationales: le cas de l'enseignement des sciences dans l'enseignement belge francophone. Éducation comparée, 7, 123-154.

Demeuse, M., Lafontaine, D. \& Straeten, M. (2005). Parcours scolaire et inégalités de résultats. In M., Demeuse, Vers une école juste et efficace: 26 contributions sur les systèmes d'enseignement et de formation: une approche internationale (pp. 259-273). Bruxelles: De Boeck.

Demeuse, M., Baye, A., Straeten, M.H., Nicaise, J. \& Matoul, A. (Éd.). (2005). Vers une école juste et efficace. 26 contributions sur les systèmes d'enseignement et de formation. Bruxelles: De Boeck.

Depover, C. \& Noël, B. (2005). Le curriculum et ses logiques. Paris: L'Harmattan.

Dumay, X. \& Dupriez, V. (Éd.). (2009). L'efficacité dans l'enseignement: Promesses et zones d'ombre. Bruxelles: De Boeck.

Dupriez, V. (2008). Les curriculums et les pratiques pédagogiques comme constructions sociales contextualisées. In F. Audigier, N. TutiauxGuillon (Éd.), Compétences et contenus. Les curriculums en questions (pp. 85-99). Bruxelles: de Boeck.

Dupriez V. \& Dumay X. (2011). Les quasi-marchés scolaires: au bénéfice de qui? Revue française de pédagogie, $\mathrm{n}^{\circ} 176,83-100$.

Duroisin, N. (2015). Quelle place pour les apprentissages spatiaux à l'école? Étude expérimentale du développement des compétences spatiales des élèves âgés de 6 à 15 ans. Thèse de doctorat (non publiée). Université de Mons.

Duroisin, N. \& Demeuse, M. (2015). What role for developmental theories in mathematics study programmes in French-speaking Belgium? An analysis of the geometry curriculum's aspects, framed by Van Hiele's model. Cogent Education, http://dx.doi.org/10.1080/233 1186X.2015.1049846. 
Duroisin, N. \& Soetewey, S. (2012, september). Give Teachers À Voice For To Change The Quality Of Current Programmes, How To Do It? With What Results? Paper session presented at the meeting of european conference on education research. Cadix, Spain.

Duroisin, N., Soetewey, S. \& Canzittu, D. (2013). On the Importance to Consider Developmental Psychology in the Process of Writing a Curriculum. Proceedings of European Conference on Education Research, Istanbul, Turquie.

Duroisin, N., Soetewey, S. \& Demeuse, M. (2012). Au carrefour du curriculum prescrit et du curriculum implanté: polémique et polysémie autour du terme de compétence en fédération Wallonie-Bruxelles. In 24 e Colloque international de l'ADMEE-Europe, Luxembourg. http://admee2012.uni.lu/pdf2012/A01_02.pdf

Duval, R. (2005). Les conditions cognitives de l'apprentissage de la géométrie: développement de la visualisation, différenciation des raisonnements et coordination de leurs fonctionnements. Annales de didactique et de sciences cognitives, vol. 10, 5-53.

Franquet, A., Friant, N. \& Demeuse, M. (2010). (S') orienter dans l'enseignement secondaire technique et professionnel en Communauté française de Belgique: la part du choix. [Version électronique]. L'Orientation Scolaire et Professionnelle, 39, (4), 507-527. Consulté le 26 décembre 2014 dans http://osp.revues.org/2937\#abstract-2937-fr

Friant, N., Demeuse, M. (2011). Un modèle du prestige des options dans l'enseignement secondaire de transition en Communauté française de Belgique». [Version électronique]. L'orientation scolaire et professionnelle, 40, (2), 183-200. Consulté le 26 décembre 2014 dans http://osp.revues.org/3099

Hirtt, N. (2005). Pédagogie de l'incompétence. Consulté le 29 août 2014 http://www.skolo. org/spip.php?article275

Houdement, C. (2007). À la recherche d'une cohérence entre géométrie de l'école et géométrie du collège. Repères, 67, 69-84.

Jonnaert, P., Ettayebi, M. \& Defise, R. (2009). Curriculum et compétences. Un cadre opérationnel. Bruxelles: De Boeck, Coll. "Perspectives en éducation \& formation».

Lafontaine, D. \& Blondin, C. (2004). Regards sur les résultats des élèves en Communauté française: apports des enquêtes de l'I.E.A., de PISA et des évaluations externes. Bruxelles, Belgique: De Boeck.

Lafontaine, D. \& Monseur, C. (2012). Entre savoirs scientifiques et usages normatifs: les enquêtes internationales d'évaluation des acquis des élèves. In L. Mottier Lopez \& G. Figari, Modélisations de l'évaluation en éducation. Questionnements épistémologiques (pp. 45-61). Bruxelles: De Boeck, Coll. «Raisons éducatives».

Legendre, M. - F. (2004). Cognitivisme et socioconstructivisme: des fondements théoriques à leur utilisation dans l'élaboration et la mise en œuvre du nouveau programme de formation. In Ph. Jonnaert et A. M'Batika, Les réformes curriculaires: Regards croisés (pp. 15-47). Québec: Presses de l'Université du Québec. 2004.

Legendre, M.-F. (2008). La notion de compétence au cœur des réformes curriculaires: effet de mode ou moteur de changements en profondeur? In F. Audigier, N. TutiauxGuillon (Éd.), Compétences et contenus. Les curriculums en questions (pp. 27-50). Bruxelles: De Boeck.

Mahoux, P. (1994). Socles de compétences dans l'enseignement fondamental et au premier degré de l'enseignement secondaire. Bruxelles: Cabinet du Ministre de l'Éducation et de l'Audiovisuel.

Malaise, S., Friant, N. \& Demeuse, M. (2014). The Equity Aspect Within the Framework of the Assessment of the Quality of Higher Education: Developing Indicators to Identify Students With a Higher Risk of Failure at University With a View to Improving Equality of Chances of Success. Revista de Docencia Universaria, 12(2), 119-141. Récupéré le 26 décembre 2015 de http://red-u.net/redu/index.php/REDU/article/view/876/pdf.

Mangez, E. (2004). La production des programmes de cours par les agents intermédiaires: Transfert de savoirs et relations de pouvoir. Revue française de Pédagogie, 146, 65-77. 
Mangez, C. \& Mangez, E. (2008). Analyse sociologique des discours pédagogiques. Application au cas de la politique éducative en Belgique francophone. In D. Frandji \& Ph. Vitale. (Éd.), Actualité de Basil Bernstein. Savoir, pédagogie et société (pp. 189-206). Rennes: Presses universitaires de Rennes.

Mathé, A.-C. (2008). Confrontation aux objets et processus de conceptualisation en géométrie à la fin de l'école primaire, rôle des interactions langagières (contribution 3). Efficacité et équité en éducation France. 1-14.

Monseur, C. \& Demeuse, M. (2004). Quelques réflexions méthodologiques à propos des enquêtes internationales dans le domaine de l'éducation. Politiques d'éducation et de formation: Analyses et comparaisons internationales, 11, 37-54.

Monseur, C. \& Lafontaine, D. (2009). L'organisation des systèmes éducatifs: quel impact sur l'efficacité et l'équité? In V., Dupriez \& X., Dumay (Éd.), L'efficacité en éducation, promesses et zones d'ombre. Bruxelles: De Boeck.

Perrenoud, Ph. (1999). Transférer ou mobiliser ses connaissances? D'une métaphore à l'autre: implications sociologiques et pédagogiques. Récupéré le 29 juin 2011 de http://www.unige. ch/fapse/SSE/teachers/perrenoud/php_main/php_1999/1999_28.html

Perrin-Glorian, M. - J., Mathé, A.-C. \& Leclercq, R. (2013). Comment peut-on enseigner la continuité de l'enseignement de la géométrie de 6 à 15 ans? Le jeu sur les supports et les instruments. Repères, 90, 5-41.

Prégent, R., Bernard H. \& Kozanitis, A. (2009). Enseigner à l'université dans une approche programme. Québec: Presse internationales polytechniques.

Roegiers, X. (2000). Une pédagogie de l'intégration: compétences et intégration des acquis dans l'enseignement. Paris-Bruxelles: De Boeck Université.

Schneider-Gilot, M. (2006). Quand le courant pédagogique «des compétences» empêche une structuration des enseignements autour de l'étude et de la classification des questions parentes. Revue française de pédagogie, 154, 85-96.

Sivesind, K.(2013). Mixed Images and Merging Semantics in European Curricula, Journal of Curriculum Studies, 45, (1), 52-66, DOI: 10.1080/00220272.2012.757807

Soetewey, S. \& Duroisin, N. (2012). Une évaluation certificative externe des compétences en sciences: mission (im)possible en Belgique francophone. In $24^{e}$ Colloque international de l'ADMEE-Europe, Luxembourg.

Soetewey, S., Duroisin, N. \& Demeuse, M. (2011). Le curriculum oublié. Analyse comparée des programmes de sciences en Belgique francophone. Revue internationale d'éducation de Sèvres, 56, 123-134.

Soetewey, S., Demeuse, M., Duroisin, N., Letor, C. \& Malaise, S. (2014). Diversité et finalités des dispositifs et outils d'évaluation des compétences. Quelle(s) cohérence(s), en Belgique francophone, dans un système initialement construit autour de la liberté d'enseignement?» in C. Dierendonck, E. Loarer \& B. Rey (Éd.), L'évaluation des compétences en milieu scolaire et en milieu professionnel (pp. 97-115). Bruxelles: De Boeck.

Usiskin, Z., Andersen, K. \& Zotto, N. (2010). Future Curricular Trends in School Algebra and Geometry: Proceedings of a Conference. Research in Mathematics in Education. Iowa State University: USA.

Van Hiele, P. M. (1986). Structure and Insight: a Theory of Mathematics Education. Orlando: Academic Press.

Tardif, J. (2006). L'évaluation des compétences. Documenter le parcours de développement. Montréal: Chenelière Éducation.

Westbury, I. (2007). Making curricula: Why states make curricula, and how. In F. M. Connelly, The SAGE Handbook of Curriculum and Instruction, Toronto: Sage, 45-65.

Mots-clés: Éfficacité, équité, curriculum prescrit, approche par compétence, communauté française de Belgique 


\section{Effizienz und Gerechtigkeit der Bildungssysteme unter dem Gesichtspunkt des vorgeschriebenen Curriculum in einer kompetenzorientierten Vorgehensweise. Der Fall des französischsprachigen Teil Belgiens}

Neben Recherchen zur Effizienz und Gerechtigkeit der Bildungssysteme im in-ternationalen Vergleich oder Studien zu den Schülerbewegungen innerhalb der Bildungssysteme ist es möglich diese beiden Schwerpunkte anhand einer Ana-lyse der Lehrplandokumente zu erfassen. Der vorliegende Artikel behandelt dieses Thema in einem besonderen Kontext, nämlich dem französischsprachi-gen Teil Belgiens. Dort besitzen die Schulprogramme zwar einen gemeinsamen Rahmen, der jedoch viel Gestaltungsspielraum gewährt. Die wichtigste hier er-örterte These geht davon aus, dass diese Gestaltungsfreiheit, sofern diese vor-handen ist, die Effizienz und die Gerechtigkeit in einem noch wenig regulierten Bildungssystem kaum begünstigt.

Schlagworte: Effizienz, Gerechtigkeit, Lehrplan, kompetenzorientierte Vorgehensweise, Französische Gemeinschaft Belgiens

\section{L'efficacià e l'equità dei sistemi scolastici confronti del curriculum prescritto in un approcio per competenze Focus sullà situazione in communità francofonà del belgio}

Attraverso di ricerche sull'efficacia e l'equità dei sistemi scolastici che utilizzano confronti statistici internazionali o lo studio dei flussi degli alunni interni ai sistemi scolastici, è possibile tenere conto di queste due grandi dimensioni analizzando i documenti curriculari. È ciò che si propone di fare questo articolo in rapporto a un contesto molto particolare: il Belgio francofono, dove i programmi di studio, sebbene siano inquadrati in un contesto comune, godono, nel complesso, di un'ampia libertà. La tesi principale sviluppata a cui ci riferiamo vuole dimostrare che questa libertà, se esiste, non è in alcun modo un fattore che favorisce l'efficacia e l'equità di un sistema scolastico ancora relativamente poco regolamentato.

Parole chiave: Efficacia, equità, curriculum prescritto, approccio per competenze, Comunità francofona del Belgio 


\title{
Efficiency and Equity of educational systems in the light of prescribed curriculum within a competency-based approach. The case of the French speaking Community of Belgium
}

\begin{abstract}
Besides research concerning the efficiency and the equity of education systems through comparisons of international statistics or the study of pupils' flows within education systems, it is possible to take into account these two main dimensions through the analysis of curricular material. This is what this article provides with in a very particular context: the French-speaking Belgium school community where curricula, although prescribed within a framework of common rules and references, allow a large degree of freedom to education networks. The main thesis developed here suggests that this freedom is probably not a factor of efficiency and equity in an educational system still weakly regulated.
\end{abstract}

Keywords: Efficiency, equity, prescribed curriculum, competency-based approach, French speaking Community of Belgium 